\title{
Timo Kost
}

\section{Transnationales Datenschutzrecht.}

\section{Am Beispiel technischer Standardisierung im Internet*}

Wenn der Vorstandsvorsitzende der Internetsuchmaschine Google, Eric Schmidt, für die Etablierung globaler Datenschutzstandards eintritt, ${ }^{1}$ dann deckt sich das mit gleichlautenden Forderungen in der rechtswissenschaftlichen Literatur. ${ }^{2}$ Allerdings bestehen schon zwischen den USA und der Europäischen Union gravierende Unterschiede in den Auffassungen über das notwendige Maß an Datenschutz. ${ }^{3}$ Ein völkerrechtlicher Vertrag mit einheitlichen Datenschutzstandards erscheint schon allein deshalb nicht realistisch.

Gleichwohl ist Datenschutz ein globales Problem und verlangt nach Lösungen, die an den Grenzen des Nationalstaats nicht Halt machen. Am Beispiel eines globalen Problems für den Datenschutz, der technischen Standardisierung im Internet, beschäftigt sich der folgende Beitrag mit den Unzulänglichkeiten der gegenwärtig geführten Modernisierungsdebatte zum Datenschutzrecht, die trotz innovativer Elemente immer noch zu stark in nationalen Dimensionen denkt, und skizziert stattdessen das Konzept eines transnationalen Datenschutzrechts.

\section{A. Technische Standards als Problem des Datenschutzes}

Im Kontext moderner Informationstechnologien, wie sie dem Internet zugrunde liegen, kann man technische Standards als eine Menge von Regeln definieren, die Bedingungen für den Austausch von Informationen zwischen Komponenten in Telekommunikations- oder Computernetzwerken festlegen. ${ }^{4}$

Gerade in heterarchisch organisierten Netzwerkumgebungen wie dem Internet erfordert der Prozess der Informationsverarbeitung ein hohes Maß an Standardisierung in Form von Kompatibilitätsstandards. ${ }^{5}$ Neben der physikalischen Kompatibilität von zwei Computern (Hardware) ist für eine „Kommunikation“ zwischen beiden auch erforderlich, dass beide die gleiche „Sprache“ sprechen, also die Prozeduren (Protokolle), nach denen beide Computer ihre Kommunikation steuern und ihre Mitteilungen formulieren, kompatibel sind. ${ }^{6}$ Im Internet

* Für Anmerkungen und Kritik danke ich Daniel Saam, Prof. Andreas Fischer-Lescano, Markus Pöcker und Prof. Ute Sacksofsky.

1 Eric Schmidt, FAZ Nr. 216 v. 17.9.2007, 19.

2 Siehe statt vieler Stein, Transnationale Datenübermittlung und die Kompetenz der Europäischen Gemeinschaft zum Abschluss eines internationalen Abkommens zum Datenschutz mit Drittstaaten, in: Arndt/Knemeyer/Kugelmann/Meng/Schweitzer (Hrsg.), FS für Walter Rudolf, 2001, S. 513 ff.

3 Reidenberg, Resolving Conflicting International Data Privacy Rules in Cyberspace, 52 Stanford Law Review (2000), 1315; Tinnefeld/Ehmann/Gerling, Einführung in das Datenschutzrecht, 4. Auflage 2005, S. $352 \mathrm{ff}$.

4 Siehe Vesting, The Autonomy of Law and the Formation of Network Standards, 5 German Law Journal (2004) 639 (644).

5 Delfs, Innovation - Standardisierung - Recht, in: Eifert/Hoffmann-Riem (Hrsg.), Innovation und rechtliche Regulierung, 2002, S. 171 (200).

6 Genschel, Standards in der Informationstechnik, 1995, S. 114. 
werden diese Standards von Standardisierungsgremien wie der Internet Engineering Task Force (IETF) oder dem WorldWideWeb-Konsortium (W3C) entwickelt.

Die IETF $^{7}$ hat selbst keine rechtliche Organisationsform und ist nur ein loser Zusammenschluss von Akteuren, denen an der Weiterentwicklung von Internetstandards gelegen ist. Diese Standards werden in sogenannten Working Groups erarbeitet, die ihre Arbeit durch Mailing Listen organisieren. Entscheidet sich die Working Group dafür, dass ein Entwurf für die Verabschiedung als Standard geeignet ist, wird von einem weiteren Gremium nach festgelegten Voraussetzungen geprüft, ob der Entwurf den Anforderungen eines IETF-Standards entspricht. Der Standard wird dann in der Publikationsserie des IETF als sogenannter Request for Comments (RFC) verabschiedet. Interessant ist dabei, dass grundsätzliche Verfahrensweisen der IETF ebenfalls in solchen RFCs festgelegt sind. So sind die Entscheidungen in den Working Groups als „rough consensus“ zu treffen, was im Rahmen des RFC 2418 festgelegt ist, der daneben zahlreiche weitere Verfahrensregeln für die Arbeitsweise in den Working Groups enthält. Solche RFC können wiederum Verweise auf andere RFCs enthalten.

Das W3 $C^{8}$ ist ein internationales Industrie-Konsortium, das 1994 gegründet wurde und für die Entwicklung und Implementierung der im WorldWideWeb verwendeten Standards verantwortlich ist. Die Ergebnisse der Arbeit des W3C werden als sogenannte „recommendation“ verabschiedet, in der ein Web-Standard beschrieben ist, der auf seine Interoperabilität mit den gängigen im Web verwendeten Hard- und Softwarekomponenten durch das W3C geprüft wurde. Diese Empfehlungen sind auf Umsetzung durch die Soft- und Hardwareindustrie angelegt, die sich so die geleistete Kompatibilisierungsarbeit zunutze machen kann. Der Entscheidungsprozess erfolgt, anders als bei der IETF, unter Ausschluss der Öffentlichkeit durch einen kleinen Kreis großer Firmen. ${ }^{9}$

Insbesondere im nordamerikanischen Schrifttum ist auf den problematischen Einfluss, den der Code auf gesellschaftliche Kommunikation im Internet ausübt, hingewiesen worden. ${ }^{10}$ Technische Standardisierung als Teilaspekt des Code trifft wesentliche Vorentscheidungen über das mögliche Nutzerverhalten im Internet. Besucht man z.B. ein Geschäft in der realen Welt, kann man sich einer Registrierung dieses Besuchs unerkannt wieder entziehen. Dagegen ermöglichen im Internet die technische Architektur, die Netzwerkprotokolle und die Software, dass ich bereits beim Aufruf der Webseite eines E-Commerce-Händlers Spuren hinterlasse. Noch bevor normative Regeln eingreifen können, ist der Schutz personenbezogener Daten im Internet also stark von der technischen Ausgestaltung abhängig.

\section{B. Modernisierung des Datenschutzrechts}

In der gegenwärtigen Debatte zu einer Reform des Datenschutzrechts taucht das globale Problem der technischen Standardisierung jedoch allenfalls am Rande

7 Zum Folgenden van Schewick, Institutionelle Grundlagen: Standardisierungsgremien und -konflikte im TK-Bereich, in: Die Rolle staatlicher Akteure bei der Weiterentwicklung von Technologien in deregulierten TK-Märkten, Studie des Deutschen Instituts für Wirtschaftsforschung im Auftrag des Bundesministeriums für Bildung und Forschung, 2006, S. $164 \mathrm{ff}$.

8 Zur Arbeitsweise des W3C siehe auch Lohse/Janetzko, Technische und juristische Regulationsmodelle des Datenschutzes am Beispiel von P3P, CR 2001, 55 ff.; Mayer, Selbstregulierung im Internet: Institutionen und Verfahren zur Setzung technischer Standards, K\&R 2000, $13 \mathrm{ff}$.

9 Lohse/Janetzko (Fn. 8), 57.

10 Prominent etwa Lessig, Code und andere Gesetze des Cyberspace, 2001. 
auf. Die Ansätze sind gegenüber der geltenden Rechtslage innovativ, jedoch zu stark an Konzepten orientiert, die in den 1990er Jahren auf Regulierungsprobleme im Nationalstaat reagierten.

\section{Strukturschutz statt individuelle Verarbeitungssituation}

RFID-Tags oder ubiquitäres Computing ${ }^{11}$ sind nur zwei Beispiele für Entwicklungen, die das überkommene Datenschutzrecht mit seinen Prinzipien wie Transparenzgebot oder Zweckbindungsgebot vor Grenzen stellen. ${ }^{12}$ Vor diesem Hintergrund vollzieht sich eine datenschutzrechtliche Reformdebatte, die die Abkehr von der individuellen Verarbeitungssituation als einzigen Ausgangspunkt für eine datenschutzrechtliche Regulierung fordert und stattdessen versucht, den Umgang mit persönlichen Daten in einem größeren Verarbeitungskontext zu sehen und mit Strukturanforderungen zu regulieren. ${ }^{13}$

Berechtigterweise erkannte man schon früh, dass dazu neben rein normativen Datenschutzkonzepten auch ein Rückgriff auf die Technologie selbst als Mittel zur Gewährleistung von Datenschutz erfolgen muss. ${ }^{14}$ „Datenschutz durch Technik“, „privacy enhancing technologies“ (PET), ${ }^{15}$ Selbstdatenschutz ${ }^{16}$ oder Systemdatenschutz ${ }^{17}$ sind die in diesem Zusammenhang in der Diskussion verwendeten Termini. Grundsätzlich kann man unterscheiden zwischen der Entwicklung von Technologien zur Ermöglichung von Datenschutz (Selbstdatenschutz, "privacy enhancing technologies“) und der Inpflichtnahme von Technikentwicklern als Adressaten von Datenschutz (Systemdatenschutz). Den Technikentwicklern sollen Prüfpflichten für eine datenschutzkonforme Ausgestaltung der Produkte, Pflichten zur Dokumentation dieser Prüfungen und Hinweispflichten für Restrisiken auferlegt werden, ${ }^{18}$ Datenschutz soll insgesamt ins „Vorfeld des Personenbezugs“ ausgedehnt werden. ${ }^{19}$ Problematischen Technologien soll durch Zertifizierung die Konformität mit den datenschutzrechtlichen Anforderungen bescheinigt werden. ${ }^{20}$

Die Tätigkeit von Akteuren wie IETF oder W3C wird in dieser Debatte nur am Rande erwähnt. Technische Internetstandards könnten jedoch genauso wie jede andere Technologie einer Prüfungspflicht hinsichtlich ihrer datenschutzkonformen Ausgestaltung unterliegen, was bislang aber nicht diskutiert wird. Insgesamt ist der gegenwärtigen Debatte ein mehr oder weniger großes Desinteresse an internationalen Standardisierungsgremien zu konstatieren.

11 Roßnagel/Müller, Ubiquitious Computing - neue Herausforderungen für den Datenschutz, CR 2004, 625 (628); Langheinrich, Die Privatsphäre im Ubiquitious Computing - Datenschutzaspekte der RFIDTechnologie, in: Fleisch/Mattern (Hrsg.), Das Internet der Dinge - Ubiquitious Computing und RFID in der Praxis, 2005, S. $329 \mathrm{ff}$.

12 Neumann/Schulz, Modernisierung des Datenschutzes: Herausforderungen durch die Technik, DuD $2007,248 \mathrm{ff}$.

13 Roßnagel, Modernisierung des Datenschutzrechts für eine Welt allgegenwärtiger Datenverarbeitung, MMR 2005, 71.

14 Zusammenfassend Simitis, Auf dem Weg zu einem neuen Datenschutzkonzept, DuD 2000, 714 (725).

15 Hansen, in: Roßnagel (Hrsg.), Handbuch Datenschutzrecht, 2003, Kapitel 3.3.

16 Roßnagel, in: Roßnagel (Hrsg.), Handbuch Datenschutzrecht, 2003, Kapitel 3.4.

17 Dix, in: Roßnagel (Hrsg.), Handbuch Datenschutzrecht, 2003, Kapitel 3.5.

18 Roßnagel (Fn. 13), 75; Roßnagel, Datenschutz in einem informatisierten Alltag, Gutachten im Auftrag der Friedrich-Ebert-Stiftung, 2007, S. 192.

19 Dix, Modernisierung des Datenschutzes: Lösungsansätze, DuD 2007, 256 (257).

20 Siehe zum Datenschutzaudit nur Roßnagel, in: Roßnagel (Hrsg.), Handbuch Datenschutzrecht, Kapitel 3.7 . 
Dieses Desinteresse hängt vornehmlich damit zusammen, dass die Debatte sich zu stark an den Denkmustern der Diskussionen der 1980er und 1990er um eine sich wandelnde Regulierungstätigkeit des Staates angesichts drängender Umweltprobleme und Probleme der wohlfahrtsstaatlichen Verrechtlichung orientiert.

\section{Risiko}

Kennzeichnend für die Debatte ist zunächst die Einführung eines Begriffs, den man eher aus anderen Rechtsbereichen wie dem Umweltrecht kennt. Gefordert wird eine risikoadäquate Umbildung des am Grundrecht auf informationelle Selbstbestimmung orientierten datenschutzrechtlichen Schutzprogramms. ${ }^{21}$ Es gelte, den Risiken neuer Technologien, deren konkrete Anwendungsfelder noch kaum zu überblicken sind, durch eine flexible Ausgestaltung des Regelungsprogramms zu begegnen.

Zwar war das Datenschutzrecht schon immer von einem an der technischen Entwicklung orientierten Regelungskonzept gekennzeichnet, allerdings greifen die neuen Konzepte unter Hinweis auf „Risiken“ grundsätzlicher in die dogmatische Struktur des Datenschutzrechts ein.

Als soziologischer Begriff bezeichnet „Risiko“ zunächst ein zeitliches Phänomen. Soziale Probleme werden vor dem Hintergrund technischen Fortschritts verstärkt zukunftsbezogen wahrgenommen. ${ }^{22}$ Man weiß zum Zeitpunkt einer Entscheidung über den Einsatz einer Technologie nicht sicher, ob oder welche negativen Auswirkungen diese haben wird, steht aber gleichzeitig unter dem Druck des gesellschaftlichen Fortschritts, durch den Nichteinsatz einer Technologie Innovation verhindert zu haben. ${ }^{23}$ Allein dieser Zukunftsbezug ist jedoch an sich nicht neu und rechtfertigt auch nicht, einen Begriff wie „Risiko“ neu einzuführen. Unsicherheit in Bezug auf künftige Schäden gab es schon immer. Was sich ändert, ist aber die Form des Umgangs mit dieser Unsicherheit. Die traditionelle Form dieses Umgangs besteht darin, einen etwaigen zukünftigen Schaden als extern veranlasst zuzuschreiben („Gefahr“). Demgegenüber werden potentielle Schäden als „Risiko“ einer Entscheidung in der Gegenwart zugerechnet. ${ }^{24}$ Der historische Grund für die Transformation des gesellschaftlichen Umgangs mit Unsicherheit vom Modus der Gefahr zum „Risiko“ liegt in der Ausdehnung der Entscheidungspotentiale in der sich immer weiter ausdifferenzierenden Gesellschaft. Der anwachsende Alternativenreichtum führt dazu, dass immer mehr Zustände als Folgen von Entscheidungen, also als „Risiko“ angesehen werden. ${ }^{25}$ „Risiko“ in diesem Sinne soll also verstanden werden als entscheidungsabhängige, abstrakte Möglichkeit eines Schadens an noch unbestimmten Rechtsgütern. ${ }^{26} \mathrm{Zu}$ diesem zeitlichen Aspekt tritt die Unmöglichkeit der $\mathrm{Zu}$ rechnung eines Schadens zu einer konkreten Technologie oder zu einem konkreten Verursacher. ${ }^{27}$ Die Tradition hatte sich demgegenüber für den Umgang mit Unsicherheit noch auf erkennbare Gesetzmäßigkeiten oder statistische Kalkulationen verlassen können. ${ }^{28}$ 
Auch die Gefahren des Internet für den Einzelnen sind mit der Risikosemantik greifbar. Als „Netzwerk der Netzwerke“29 ist das Internet durch eine relationale Verknüpfung seiner Elemente gekennzeichnet. Diese Logik und die damit einhergehende rasante technologische Entwicklung verhindern, dass sich stabile Formen des Wissens um die Folgen von Entscheidungen über den Einsatz dieser Technologien herausbilden können. ${ }^{30}$

\section{Regulierte Selbstregulierung}

Die methodischen Schlussfolgerungen aus dieser Einführung der Risikokategorie in die Debatte um die Reform des Datenschutzes vollziehen sich dagegen in vorsichtigeren Bahnen. Die Konzepte des Selbstdatenschutzes und Systemdatenschutzes sind der Einsicht geschuldet, dass man mit den zentralen Begriffen der verantwortlichen Stelle ( $\int 2$ BDSG) und der Verarbeitung personenbezogener Daten ( $\int 3$ Abs. 4 BDSG) als regelungsauslösenden Vorgang an Grenzen stößt. ${ }^{31}$ Die Adressatenstellung und die eine Regulierung auslösende Situation wird durch System- und Selbstdatenschutz gegenüber den tradierten Datenschutzkonzepten weiter ins Vorfeld verlagert.

Das ähnelt der Reaktion der Diskussion der späten 70er und frühen 80er Jahre im Umwelt- und Technikrecht, die eine Abkehr vom polizeirechtlich geprägten Ordnungsrecht mit seinen zentralen Figuren des Störers und der Gefahr hin zu einer den Bedingungen der modernen „Risikogesellschaft“ entsprechenden Rationalität mit Begriffen wie dem Vorsorgeprinzip forderte. ${ }^{32}$ Das Recht sollte „prozedural“ ${ }^{33}$ werden, indem es unter anderem materielle Letztentscheidungskompetenzen über Generalklauseln wie den „Stand der Technik“ (z.B. \3 Abs. 6 BImSchG) in gesellschaftliche Bereiche wie das Wissenschaftssystem externalisiert; gleichzeitig sollten jedoch durch prozedurale Elemente Defizite der Wissenschaft im Umgang mit dieser Verantwortung ausgeglichen werden, die daraus resultieren, dass die Funktionslogik der Wissenschaft nicht auf den allgemeinwohlverträglichen Ausgleich gesellschaftlicher Partikularinteressen ausgerichtet ist. ${ }^{34}$ Die Abkehr vom Begriff des Störers und der Gefahr, die dem Verschwinden sicherer Kausalzusammenhänge und damit individualisierbarer Gefährdungslagen geschuldet sein sollten, war die zweite große Forderung in diesem Zusammenhang. ${ }^{35}$ Diese Überlegungen bereiteten sodann den Weg für die in den 90er Jahren begonnene Diskussion um „Regulierte Selbstregulierung“ als Steuerungskonzept einer fragmentierten Gesellschaft ohne Zentrum und ohne Spitze, in welcher der Gewäbrleistungsstaat ${ }^{36}$ unter Ungewissheitsbedingungen in zunehmendem Maße abhängig von einem nicht mehr allgemein zugänglichen Wissen ist.

Die Forderung nach Strukturschutz im Vorfeld des Personenbezugs (oben B.I.) ist vor diesem Hintergrund als der regulierten Selbstregulierung ähnliches Konzept zu verstehen. Ihr Schutzprogramm ergänzt zentrale Begriffe des Daten-

Noam, Beyond Liberalization: From the Network of Networks to the System of Systems, in: Hoffmann-Riem/Vesting (Hrsg.), Perspektiven der Informationsgesellschaft, 1995, $49 \mathrm{ff}$.

30 Ladeur, Datenschutz - vom Abwehrrecht zur planerischen Optimierung von Wissensnetzwerken, DuD 2000, 12 (18); Vesting (Fn. 4), 657.

31 Roßnagel (Fn. 13), 74.

32 Wolf, Zur Antiquiertheit des Rechts in der Risikogesellschaft, Leviathan 1987, $357 \mathrm{ff}$.

33 Grundlegend Teubner, Reflexives Recht, ARSP 1982, 13; aus der Diskurstheorie Habermas, Faktizität und Geltung, Kapitel IX, insbes. S. $516 \mathrm{ff}$.

34 Wolf (Fn. 32), $373 \mathrm{ff}$.

35 Ebd.

36 Franzius, Der "Gewährleistungsstaat" - ein neues Leitbild für den sich wandelnden Staat?, Der Staat 42 (2003), $493 \mathrm{ff}$. 
schutzrechts (verantwortliche Stelle, Verarbeitung personenbezogener Daten)

und sichert ihre Legitimation über die Inanspruchnahme des Staats als Letztgaranten (Prüfpflichten für Technikgestaltung).

\section{Kritik}

\section{Die Stellung des Staates}

Ausgangspunkt der Kritik ist der etatistische Reduktionismus der beschriebenen Reformvorschläge, der die tatsächlichen Probleme der Eigendynamik einer gesellschaftlichen Rationalität wie der Technologie auf globaler Ebene nicht ausreichend thematisieren kann. Die vorgestellten Lösungsstrategien verbleiben einer Theorie des Gewährleistungsstaats verhaftet, die immer noch den Staat als Letztgarant des Gemeinwohls ansieht. ${ }^{37}$ Der private Sektor wird in ein so konstruiertes Konzept nur als genuin gemeinwohlgefährdender Akteur integriert, dessen prinzipiell destruktiven Tendenzen es aus einer übergeordneten staatlichen Perspektive entgegenzuwirken gelte. Damit wird dem Staat eine Supervisions-Kompetenz und vor allem eine Supervisions-Fähigkeit zugeschrieben, die er unter modernen Bedingungen der Komplexität einer ausdifferenzierten Gesellschaft auf globaler Ebene nicht mehr hat. „Datenschutz durch Technik“ wird erst mit der Einsicht plausibel, dass der Staat auch in Fragen des Datenschutzes nicht mehr in der Lage ist, ausreichendes Wissen über regulierungsrelevante Zusammenhänge zu akkumulieren und aus diesem Grund auf Expertenwissen der Technikdesigner angewiesen ist. Damit reihen sich solche Konzepte zwar ein in Formen der Kooperation des Staates mit anderen Akteuren, die im Einzelfall vielleicht auch Erfolg haben mögen. Methodisch gesehen stützen solche Kooperationsverhältnisse allerdings gesellschaftliches Handeln mehr oder weniger unreflektiert $a b .{ }^{38}$ So ist für „Datenschutz durch Technik“ völlig unklar, wie die bloße Förderung datenschutzfreundlicher Technologien einen auch nur einigermaßen abgrenzbaren Beitrag zu einer Ordnungsidee liefern könnte.

Kann die massive normative Relevanz von nicht-staatlich gesetzten Normen oder Standards im nationalen Bereich noch durch einen re-entry ${ }^{39}$ über Scharnierbegriffe wie „Stand der Technik“ mehr oder weniger erfolgreich verdeckt und dadurch in die klassische Rechtsquellenlehre integriert werden, ${ }^{40}$ versagt diese Strategie auf der internationalen Ebene insofern, als den meisten Standardisierungsgremien eine völkervertragsrechtliche Basis fehlt. Eine solche Strategie würde darüber hinaus auch der Tatsache widersprechen, dass die Abhängigkeit des „Supervisions-Staates“ von Expertenwissen im Bereich des Internets - und hier vor allem der technischen Standardisierung - massiv gestiegen ist. Staatliche Überwachung der technischen Standardisierung als einzige Alternative würde - in tradierter Sichtweise - nur aus der Perspektive eines Weltstaates Sinn machen, der eine dem Nationalstaat entsprechende Zugriffsmöglichkeit auf den regulierten Sozialbereich haben müsste. Wer vermag schon - angesichts des Erfordernisses komplexen Technikwissens - konkrete und detaillierte normative Anforderungen an eine Internettechnologie zu stellen?

39 George Spencer Brown, Laws of Form, 1969, deutsche Ausgabe: Gesetze der Form, 1997; bezogen auf das Rechtssystem Luhmann, Das Recht der Gesellschaft, 1993, S. 76.

40 Pöcker, Stasis und Wandel der Rechtsdogmatik, 2007, S. $118 \mathrm{ff}$. 
Die in Mode gekommene Verwendung des Begriffs „transnationales Recht“41 deutet auf die Zunahme globaler Prozesse jenseits der internationalen Politik hin und fordert unser Nachdenken über die Rolle des Staates und des Rechts in einer sich wandelnden Gesellschaft in gleichem Maße heraus wie die Beschreibungsformel der „Risikogesellschaft“. In den 80er Jahren führte die Semantik der „Risikogesellschaft“ zur Suche nach Konzepten über eine veränderte Rolle des Staates in Fragen des Gemeinwohls. Heute kennzeichnet die Debatte um „transnationales Recht“ die Suche nach dem Erhalt der Funktion des Rechts, normative Erwartungen in einer Welt zu stabilisieren, die gerade auf der globalen Ebene so wenig durch Recht determiniert zu sein scheint. Neben der Umstellung auf die Beobachtung moderner Informationstechnologien unter dem Leitbegriff „Risiko“ muss die Reformdebatte im Datenschutzrecht vor diesem Hintergrund noch einen weiteren Aspekt bedenken, den der Risikobegriff allein nicht fassen kann: die zunehmende transnationale Regulierung von Sachverhalten durch private Akteure.

Zwar adressiert die neuere Reformdebatte Technikentwickler als Verantwortliche von Datenschutzkonformität, entsprechend dem Territorialitätsprinzip stammen die Prinzipien, auf welche sie verpflichtet werden sollen, jedoch notwendiger Weise aus dem nationalen Recht. Die Entwicklung von technischen Standards durch globale Standardisierungsgremien würde sich dann den Anforderungen einer Vielzahl von regionalen Datenschutzkonzepten ausgesetzt sehen. In Konkurrenz treten hierbei nicht bloß die unterschiedlichen Konzepte der USA und der Europäischen Union, sondern auch andere regionale Ausdifferenzierungen, wie etwa das Privacy-Framework der Asia-Pacific Economic Cooperation (APEC). ${ }^{42}$ Erklärt man einen solchen Wettbewerb zum Konzept mit dem Ziel „gemeinsamer" Wertentscheidungen über Fragen von informationeller Selbstbestimmung, ${ }^{43}$ setzt man zu einseitig auf staatliche Instanzen und verkennt die Realität eines durch private Akteure dominierten transnationalen Internets. Bleibt man beim Risikobegriff als einziger Beschreibung gesellschaftlicher Realität stehen, kann man die massive normative Relevanz privater technischer Standardsetzung im Internet nicht fassen. Im selben Maße müssen Konzepte regulierter Selbstregulierung die prinzipiell fortexistierende Fähigkeit des States zu ordnungsrechtlichem Handeln im zu regulierenden Bereich unterstellen. ${ }^{44}$ Das setzt aber einen für staatliche Interventionen verfügbaren gesellschaftlichen Handlungszusammenhang voraus, in dem notfalls einzelne Akteure flankierend adressiert werden können. ${ }^{45}$ Globale, nicht auf völkerrechtlichen Verträgen beruhende technische Standardisierungsgremien entziehen sich dieser Voraussetzung jedoch weitgehend.

41 Harold Hongjuh Koh, Transnational Legal Process, 75 Nebrasca Law Review 181 (1996); Jessup, Transnational Law (Storrs lectures on jurisprudence), 1956. Für das Umweltrecht Herberg, Erzeugen multinationale Unternehmen ihr eigenes Umweltrecht?, in: Winter (Hrsg.), Die Umweltverantwortung multinationaler Unternehmen. Selbststeuerung und Recht bei Auslandsdirektinvestitionen, 2005, S. 73, sowie Fischer-Lescano, Transnationales Verwaltungsrecht, JZ 2008, 373.

42 http://www.apec.org/apec/apec_groups/som_special_task_groups/electronic_commerce.html (abgerufen: 25.3.2009).

43 Ausdrücklich Roßnagel, Weltweites Internet - globale Rechtsordnung?, MMR 2002, 67 (70).

44 Kloepfer (Fn. 37), \4 Rn. $51 \mathrm{ff}$. Zu Auflösungserscheinungen der staatlichen Zugriffsmöglichkeit im nationalen Bereich Hoffman-Riem, Governance im Gewährleistungsstaat - Vom Nutzen der Governance-Perspektive für die Rechtswissenschaft, in: Schuppert (Hrsg.), Governance-Forschung. Vergewisserung über Stand und Entwicklungslinien, 2005, S. 195, $213 \mathrm{ff}$.

45 Vgl. Pöcker, Das "Duale System”: Regulierte Selbstregulierung oder autonome Selbstorganisation? Zu angemessenen theoretischen Deutungsformen eines umweltrechtlichen Phänomens, Ancilla Juris 2007, 23. 
Doch die Perspektive ist nochmals zu erweitern. Soweit man sich für den internationalen Bereich auf die Logik eines Wettbewerbs konkurrierender nationaler Regulierungsstrategien zurückzieht, verkennt man, dass die Kollision von unterschiedlichen politisch-rechtlichen Auffassungen auf internationaler Ebene nur ein Oberflächenphänomen ist. ${ }^{46}$ Der tiefere Grund für das Auftauchen von Datenschutzproblemen auf internationaler Ebene sind vielmehr die Expansionstendenzen der operativ geschlossenen Funktionssysteme der Weltgesellschaft selbst. ${ }^{47}$ Im transnationalen Raum des Internets kollidieren und konkurrieren in erster Linie nicht autonome nationalstaatliche Normmassen von national-staatlichen Akteuren, sondern unterschiedliche gesellschaftliche Rationalitäten: Informationelle Selbstbestimmung vs. technische Internetstandards (P3P), Informationelle Selbstbestimmung vs. wirtschaftliche Logik (E-Commerce), Informationelle Selbstbestimmung vs. staatliche Sicherheitsinteressen (FluggastdatenAffäre). ${ }^{48}$ Deutlichstes Zeichen dafür ist der Trend zu einer immer stärkeren Bereichsdifferenzierung des Datenschutzrechts. ${ }^{49}$

Dementsprechend wird es auch kein identifizierbares Forum auf weltgesellschaftlicher Ebene für die Beobachtung internationaler Entwicklungen geben. ${ }^{50}$ Datenschutzrechtliche Fragen werden nicht in einem abgrenzbaren internationalen Forum diskutiert und entschieden, sondern werden heute schon in einer Vielzahl von Arenen artikuliert. ${ }^{51}$ Im Bereich des geistigen Eigentums diskutiert die World Intellectual Property Organization (WIPO) datenschutzrechtliche Aspekte der Verwendung von Rights Management Information (RMI). Bei der Internet Corporation for Assigned Names and Numbers (ICANN) wird thematisiert, welche Datenschutzrelevanz die sogenannten WHOIS-Abfragen bezüglich der Inhaberschaft von registrierten Domains haben. Auch im Bereich des E-Commerce ist es eher realistisch, dass datenschutzrechtliche Fragen in verbraucherschutzrechtliche Regimes eingebunden werden, als noch von einem übergreifenden Datenschutzkonzept reguliert zu werden. ${ }^{52}$

Auf den hier untersuchten Gegenstand übertragen bedeutet das, dass es das Problem der destruktiven expansiven Tendenzen eines institutionell in Standardisierungsgremien verankerten Technikdiskurses zu lösen gilt. Technikdiskurse haben die Tendenz, ihre eigene partielle Rationalität zu universalisieren und dabei in Bereiche in ihrer Umwelt auszugreifen, worauf man z.B. mit der Entwicklung des Rechts auf informationelle Selbstbestimmung reagierte. ${ }^{53}$ Die technische Architektur, verkörpert durch technische Standardisierung, ermöglicht erst bestimmte Kommunikationen im Internet, die zu einer Gefährdung des Datenschutzes führen. Statt auf die Durchsetzung eines gemeinsamen und bereichsübergreifenden Datenschutzes auf globaler Ebene zur rechtlichen Domestizierung dieser expansiven Tendenzen der Standardisierung zu vertrauen, sollte ein Regulierungsansatz auf die eben skizzierten multipolaren Kollisionslagen einge-

Fischer-Lescano/Teubner, Regime-Kollisionen, 2006, S. $28 \mathrm{f}$.

Luhmann, Die Weltgesellschaft, in: Luhmann, Soziologische Aufklärung, Bd. 2, 5. Auflage 2005, S. 51 ff.; speziell zum Rechtssystem der Weltgesellschaft Luhmann (Fn. 39), S. 550 ff., insbes. 571 ff.; Teubner, Altera pars Audiatur, ARSP Beiheft 65 (1996), S. $199 \mathrm{ff}$.

Zur Kollision gesellschaftlicher Rationalitäten Fischer-Lescano/Teubner (Fn. 46), S. 25 ff.

Dazu Burkert, in: Roßnagel (Hrsg.), Handbuch Datenschutzrecht, 2003, Kapitel 2.3.

Ebd.

Reidenberg (Fn. 3), 1357 ff.

Z.B. die Initiative Global Business Dialogue on Electronic Commerce, http://www.gbd-e.org (abgerufen: 25.3.2009).

Albers, Informationelle Selbstbestimmung, 2005, S. 98 ff.; Roßnagel/Wedde/Hammer/Pordesch, Digitalisierung der Grundrechte?, 1990. 
stellt sein und die Abstimmung kollidierender Rationalitäten auf weltgesellschaftlicher Ebene zum Thema machen. Das erfordert, sich stärker als bisher von der Vorstellung von Datenschutz als bereichsübergreifendem Konzept zu lösen.

\section{Recht und technische Standardisierung}

Zum Abschluss soll die Idee eines transnationalen Datenschutzrechts als Kollisionsrecht entfaltet werden, das für das Problem des Verhältnisses zwischen Recht und technischer Standardisierung eine Abstimmung der nationalen Verwaltungsordnungen mit Normierungsprozessen durch vornehmlich private Akteure auf globaler Ebene sucht. In der Literatur wird zum Teil grundsätzlich bezweifelt, dass internationale Standardsetzung durch private Akteure neue theoretische Probleme schafft, weil auch schon auf der Ebene des Nationalstaats im Verhältnis von Recht und Technik die gleichen, vor allem legitimatorischen Probleme auftauchen. ${ }^{54}$ Daran ist richtig, dass die zunehmende gesellschaftliche Relevanz von Technik schon in der anti-modernistischen Debatte der Weimarer Republik $^{55}$ thematisiert wurde. In gleichem Maße wird die Abhängigkeit staatlichen Entscheidens von Expertenwissen und der damit implizierte politische Aspekt jeder Entscheidung über den Einsatz und die Ausgestaltung von Technik schon in der konservativen Kritik von Ernst Forsthoff angesprochen. ${ }^{56}$ Auch in der Folgezeit mangelte es im rechtswissenschaftlichen Schrifttum nicht an Versuchen, den Einfluss von Technik auf das Recht zu beschreiben. ${ }^{57}$ Im Unterschied dazu sehen wir uns aber heute neuen theoretischen Problemen der transnationalen Standardisierung ausgesetzt, weil ein Regulierungsansatz hier auf einem schon in hohem Maße vorab standardisierten Feld aufsetzen muss. ${ }^{58}$ Das ist für das Verhältnis zwischen staatlichem Recht und der zwischen der Mitte des 19. Jahrhunderts und dem Ersten Weltkrieg sich herausbildenden technischen Standardisierung anders. Zwar ist in diesem Zeitraum sowohl die Entstehung einer parlamentarischen Gesetzgebung, die auf völlig neuartige Phänomene der Technologie reagiert, als auch die Zunahme von technischen Regeln, die im gesellschaftlichen Bereich von den technisch-wissenschaftlichen Vereinen gesetzt werden, zu beobachten. ${ }^{59}$ Anders als die technische Standardisierung im Internet, die sich heute hauptsächlich fernab von staatlicher Regulierung vollzieht, sind die Ebenen der technischen Regeln und der staatlichen Regulierung von technischen Phänomenen in der industriellen Revolution um 1900 eng miteinander verflochten und beeinflussen sich wechselseitig. ${ }^{60}$ Als „Ordnungsmuster“61 führt eine

54 Lepsius, Standardsetzung und Legitimation, in: Möllers/Voßkuhle/Walter(Hrsg.), Internationales Verwaltungsrecht: eine Analyse anhand von Referenzgebieten, 2007, S. 345 (373).

55 Schmitt, Theodor Däublers „Nordlicht“. Drei Studien über die Elemente, den Geist und die Aktualität des Werkes, 1916 (Neuauflage 1991); Schmitt, Römischer Katholizismus und politische Form, 2. Auflage 1925. Dazu John P. Mc Cormick, Carl Schmitt's Critique of Liberalism. Against Politics as Technology, 1999. Siehe auch Breuer, Anatomie der konservativen Revolution, 1993, S. 70 ff. Aus der Nachkriegszeit Carl Schmitt, Theorie des Partisanen, 1963.

56 Forsthoff, Der Staat der Industriegesellschaft, 2. Auflage 1971; Forsthoff, Lehrbuch des Verwaltungsrechts, 9. Auflage, 1966, S. 72 ff.; Forsthoff, Rechtsstaat im Wandel, 1964, S. $197 \mathrm{ff}$.

57 Statt vieler Winner, Autonomous Technology: Technics-out-of-control as a theme in political thought, 1977. Umfassend Marburger, Die Regeln der Technik im Recht, 1979.

58 Ladeur, Die Internationalisierung des Verwaltungsrechts: Versuch einer Synthese, in: Möllers/Voßkuhle/Walter (Fn. 54), S. 388.

59 Vec, Recht und Normierung in der Industriellen Revolution. Neue Strukturen der Normsetzung in Völkerrecht, staatlicher Gesetzgebung und gesellschaftlicher Selbstnormierung, 2006, S. 165 ff. und S. $293 \mathrm{ff}$.

60 Vec (Fn. 59), S. 384 f.

61 Zum Begriff Zumbansen, Ordnungsmuster im modernen Wohlfahrtsstaat, 2000. 
„reflektierte Regulierungspraxis, die die Fortschritte von Wissenschaft und

Technik beobachtet und deren gesellschaftliches Wirksamwerden abwartet, “62 überhaupt erst zur Herausbildung von Standardisierungen.

Unsere Konsequenz daraus ist die Entwicklung eines transnationalen Datenschutzrechts, das als Kollisionsrecht neben der Erweiterung des tradierten Rechtsquellenbegriffs um die Anerkennung von Rechtsentstehungsprozessen jenseits des Staates (I.) vor allem für die Kompatibilisierung der nationalen Verwaltungsordnungen mit transnationalen Normierungen und den Einbau von umweltadäquater Reflexivität in diese Prozesse zu sorgen hat (II.).

\section{Problematik der Rechtsquellen}

Die tradierte staatszentrierte Rechtsquellenlehre muss privaten Norm- und Standardsetzungen notwendigerweise die Anerkennung als Recht versagen. ${ }^{63} \mathrm{Im} \mathrm{Na}-$ tionalstaat mag das noch plausibel sein, weil dort die normative Relevanz privater Normierungen erfolgreich durch ihre Einbindung und Disziplinierung im staatlichen Recht verdeckt werden konnte. ${ }^{64}$ Verträge zwischen global agierenden Unternehmen, interne Regelsetzungen in internationalen Organisationen, globale Standardisierung in Organisationen wie ISO, ILO oder den oben genannten Internetstandardisierungsgremien entziehen sich aber den rechtlichen Rahmenbedingungen des Nationalstaats, ohne dass funktionale Äquivalente in Sicht wären. ${ }^{65}$ Aus der hier vertretenen Perspektive, die skeptisch gegenüber allen Versuchen ist, auf weltgesellschaftlicher Ebene den Gedanken der Rechtseinheit mit hierarchisch abgestuften Normmassen und einem politischen Zentrum an der Spitze fortzusetzen, sind indes rechtspluralistische Ansätze die einzige Möglichkeit, den globalen Technikdiskurs mit real beobachtbaren Normierungen und vor allem demokratietheoretischen Defiziten unter Konstitutionalisierungsdruck zu setzen.

\section{Die normative Qualität von Netzwerk-Standards: status quo}

Während im deutschen Schrifttum noch streng zwischen Recht und Technik unterschieden wird, ${ }^{66}$ beginnt sich unter nordamerikanischen Internetjuristen eine solche Debatte über die normative Qualität von Netzwerk-Standards abzuzeichnen. Die Auffassungen lassen sich in drei Kategorien einteilen. ${ }^{67}$

Eine in der staatszentrierten Tradition stehende Meinung sieht Recht immer als Instrument der Regulierung sozialer Beziehungen an und wendet den Rechtsbegriff nur auf den Bereich menschlicher Kommunikation und Interaktion an. Deshalb sei das Konzept von Recht nicht übertragbar auf technische Standards und zwischen technischen Standards auf der einen und Rechtsnormen auf der anderen Seite strikt zu unterscheiden. ${ }^{68}$

62 Vec (Fn. 59), S. 381.

63 Ruffert, Rechtsquellen des Verwaltungsrechts, in: Hoffmann-Riem/Schmidt-Aßmann/Voßkuhle (Hrsg.), Grundlagen des Verwaltungsrechts, Band I, 2006, \$17 Rn. 18 m.w.N.; deutlich, einen sanktionsgestützten Rechtsbegriff benutzend, auch Röhl, Internationale Standardsetzung, in: Möllers/ Voßkuhle/Walter (Fn. 54), S. 319 (321).

64 Entweder als statische Verweisung, z.B. $\$ 7$ Abs. 5 BImSchG, oder als normkonkretisierende gleitende Verweisung (Stand der Technik), z.B. $\$ 3$ Abs. 6 BImSchG. Siehe zusammenfassend zu den Anerkennungsmodi Ruffert (Fn. 63), Rn. 89 ff. Weiterführend Pöcker (Fn. 40), S. 118 ff.

65 Teubner, Privatregimes: Neo-Spontanes Recht und duale Sozialverfassungen in der Weltgesellschaft, in: Simon/Weiß (Hrsg.), Zur Autonomie des Individuums: Liber Amicorum Spiros Simitis, 2000, S. $437 \mathrm{ff}$.

66 Siehe statt vieler Bendrath, Privacy Self-Regulation and the changing role of the state - from public law to social and technical mechanisms of governance, TransState Working Papers No. 59, 2007.

67 Siehe dazu Vesting (Fn. 4), $645 \mathrm{ff}$.

68 Joseph H. Sommer, Against Cyberlaw, 15 Berkeley Technology Law Journal 1145, 1151 (2000). 
Stark von tradierten Vorstellungen abstrahierend klassifiziert Reidenberg die klassisch staatliche Rechtsetzung und die Entwicklung von technischen Standards als „overlapping rule systems“, 69 die sich entweder gegenseitig ergänzen, oder, unter gewissen Umständen, substituieren. Abweichend vom klassischen Rechtsquellenbegriff ist die Quelle des Regelentstehungsprozesses die Technik selbst und der soziale Prozess, in dem gewohnheitsmäßige Praxis entsteht. ${ }^{70} \mathrm{Ge}-$ genüber dem ersten Ansatz verneint diese Meinung zwar nicht die Rolle von staatlicher Rechtsetzung als sekundär abstützenden Prozess, hat aber einen analytischen Primat auf Technik.

Eine dritte Meinung bewegt sich eng zum Ansatz Reidenbergs, gibt allerdings die Unterscheidung zwischen technischen Standards und Rechtsnormen völlig auf („CODE is Law“). Technische Standards seien nicht neutral, sondern immer eingebettet in Machtstrukturen und müssten deshalb Objekt politisch-rechtlicher Entscheidungsfindung werden. Gefordert wird hier eine Politisierung des CODE derart, dass Technische Standards in verfassungsrechtlich abgestützte Fundamentalnormen eingebettet werden müssten. ${ }^{71}$

Ein Umweg durch die theoretischen Prämissen eines systemtheoretischen Rechtsbegriffs wird zeigen, dass diese Versuche, die normative Qualität technischer Standards zu beschreiben, nicht ausreichend zwischen Normativität und bloßer Verhaltensregulierung durch Standards unterscheiden.

\section{Recht versus Nicht-Recht}

Wir nähern uns dem Problem der Rechtsqualität von technischen Standards von einem systemtheoretischen Rechtsbegriff aus. Die theoretische Begründung, unter welchen Voraussetzungen man bei privaten Regelsetzungen von Recht sprechen sollte, ist ausführlich in der juristischen Systemtheorie geleistet, wie sie insbesondere von Gunther Teubner vertreten wird. ${ }^{72}$ Ausgangspunkt ist die explizite Aufgabe der Annahme, ein Rechtssystem bestehe nur auf der nationalen Ebene. Stattdessen entwickelt sich auch das Recht, der Logik der funktionalen Differenzierung folgend, auf globaler Ebene zu einem einheitlichen Sozialsystem, dessen Einheit sich nur noch in der Bezugnahme auf den binären Code Recht/Unrecht zeigt. ${ }^{73}$ Die kommunikative Bezugnahme auf den Rechtscode allein reicht aber noch nicht aus, um von Recht zu sprechen. Der systemtheoretische Rechtsbegriff von Luhmann ist anspruchsvoller. Erst wenn sekundäre Normen institutionalisiert sind, welche die vorausgehende Beurteilung ihrerseits nach dem binären Rechtscode beobachten, ähnlich den secondary rules im Sinne von H.L.A. Hart, welche die Entstehungsvoraussetzungen für Primärnormen regeln, lässt sich von Recht sprechen. ${ }^{74}$

69 Reidenberg, Lex Informatica: The Formulation of Information Policy Rules through Technology, 76 Texas Law Review 553 (554, 566).

70 Reidenberg (Fn. 69), 566.; ähnlich Ladeur, Rechtliche Regulierung von Informationstechnologien und Standardsetzung, CR 1999, 395 (398).

71 Lessig (Fn. 10).

72 Teubner, Globale Bukowina - Zur Emergenz eines transnationalen Rechtspluralismus, Rechtshistorisches Journal 1996, 255; ferner Fischer-Lescano, Globalverfassung: die Geltungsbegründung der Menschenrechte, 2005, Kapitel 3.

73 Luhmann (Fn. 39), S. 571 ff. Zum Code Recht/Unrecht als spezifische Unterscheidung rechtlicher Kommunikationen Luhmann (Fn. 39), S. $165 \mathrm{ff}$.

74 Luhmann (Fn. 39), S. 61; Fischer-Lescano/Teubner, Fragmentierung des Weltrechts: Vernetzung globaler Regimes statt etatistischer Rechtseinheit, in: Albert/Stichweh (Hrsg.), Weltstaat und Weltstaatlichkeit: Beobachtungen globaler politischer Strukturbildungen, 2007, S. 37 ff. 
Als Beispiele für solche nicht-staatlichen Normmassen, denen Rechtsqualität zugesprochen wird, werden üblicherweise die lex mercatoria des internationalen Wirtschaftsverkehrs und die lex digitalis des Internets genannt. ${ }^{75}$

$\mathrm{Ob}$ man bei den die Architektur des Internets bildenden technischen Standards in diesem Sinne von Recht sprechen kann, erscheint indes auch unter der hier vertretenen Ansicht fraglich. Die faktische Verhaltensregulierung im Internet durch solche privat gesetzten Standards allein reicht nicht aus, um von „CODE is Law “ zu sprechen, wie dies als prominentes Beispiel Lawrence Lessig tut. ${ }^{76}$ Zwar fordert der hier vertretene Rechtsbegriff die klassische Rechtsquellenlehre in hohem Maße heraus und erlaubt grundsätzlich, Recht nicht nur in den Fällen anzunehmen, in denen mittels Legitimationsketten an politische Souveränität rückgebundene Akteure handeln; gleichwohl wird hier nicht für die Anwendung eines bis zur Unkenntlichkeit aufgeweichten Konzepts von Normativität votiert. $^{77}$

\section{a) Verwendung des Rechtscodes bei der Entwicklung technischer Standards}

Als Ausgangspunkt ist zu konstatieren, dass die Implementierung eines technischen Standards an sich noch keine Beobachtung eines Verhaltens unter dem binären Rechtscode ist. Durch einen technischen Standard wird zunächst nur das mögliche Verhalten im Internet reguliert. Verhaltensregulierung ist aber nicht das konstituierende Merkmal rechtlicher Kommunikation, sondern die Bezugnahme auf den Code Recht/Unrecht. ${ }^{78}$ Solche Standards dürfen also nicht per se als Recht missverstanden werden, vielmehr ist stärker danach zu differenzieren, ob im Laufe ihres Entwicklungsprozesses auch nach dem binären Rechtscode kommuniziert wird. Solche rechtlichen Kommunikationen unterscheiden sich deutlich von den einer technischen Rationalität folgenden Kommunikationen. Diese Unterscheidung wird von der soeben skizzierten Diskussion um die normative Qualität von Netzwerkstandards nicht deutlich genug getroffen. Für den weitaus größten Teil technischer Standards wird man wohl keine rechtlichen Kommunikationen feststellen können. Vorherrschend ist das Diktum der Interoperabilität und der Zukunftsoffenheit.

Allerdings sind bei der Entwicklung technischer Internetstandards in jüngerer Zeit immer wieder kommunikative Bezugnahmen auf den Rechtscode zu beobachten. Als Beispiel für eine solche neben der technischen Entwicklung mitlaufende Verwendung des Rechtscodes kann P3P dienen, ein technischer Standard, der vom bereits erwähnten W3C entwickelt wurde. Als klassischer Anwendungsfall sogenannter Privacy Enhancing Technologies (PET) hat P3P insbesondere im datenschutzrechtlichen Schrifttum besondere Aufmerksamkeit erfahren. ${ }^{79}$ P3P ermöglicht es dem Internetnutzer, bis zu einem gewissen Maße selbst über die Verwendung persönlicher Daten beim Aufruf einer Webseite zu entscheiden. Es handelt sich dabei um eine Protokoll-/Architektur-Kombination, die entwickelt wurde, um Webnutzer über die Verfahrensweise der Datensammlung von Webseiten zu informieren. Die P3P-Technologie ist heute in den

$\mathrm{Zu}$ lex mercatoria siehe nur Zumbansen, Lex Mercatoria: Zum Geltungsanspruch transnationalen Rechts, RabelsZ 2003, 637 ff.; zur lex digitalis des Internets Teubner, Globale Zivilverfassungen, ZaöRV 2003, $1 \mathrm{ff}$.

76 Lessig (Fn. 10).

77 Zurückhaltend bei der Frage nach der Rechtsqualität technischer Internetstandards auch Vesting (Fn. 4), 648 ff.

78 Die Verhaltensregulierung noch als Funktion des Rechts beschreibend Luhmann, Rechtssoziologie, 2. Auflage 1983. Später beschränkt sich Luhmann dann auf „kontrafaktische Stabilisierung normativen Erwartens“, Luhmann (Fn. 39), S. 131 ff.

79 Lohse/Janetzko (Fn. 8), 55. 
gängigsten Internetbrowsern (Microsoft Internet Explorer, Mozilla Firefox) integriert und ermöglicht in der derzeitigen Version 1 Einstellungen zur Verwendung von Cookies. Dem Browser-Nutzer ermöglicht der Standard, die Details zur Verwendung von Cookies durch eine Webseite zu steuern. So lassen sich beispielsweise Cookies von Webseitenanbietern blockieren, die keine Datenschutzrichtlinie haben. Die Webseiten-Betreiber ihrerseits müssen, um mit ihrer Webseite mit einem P3P-fähigen Browser Informationen über die Datennutzung austauschen zu können, Datenschutzrichtlinien aufstellen, die den Vorgaben des P3P Standards entsprechen (Angaben zur Art der Cookies, der Verweildauer auf dem Rechner des Nutzers etc.). Vor dem Abruf einer Webseite ruft der Browser des Nutzers diese Datenschutzrichtlinie des Webseiten-Betreibers ab und vergleicht sie mit den Einstellungen des Nutzers. Bei Abweichungen von den Nutzer-Einstellungen oder wenn ein Webseiten-Betreiber keine P3P-Datenschutzrichtlinie verwendet, gibt der Internet-Browser vor dem Laden der Seite einen Warnhinweis, so dass der Nutzer selbst entscheiden kann, ob das Laden fortgesetzt oder abgebrochen werden soll.

Ganz deutlich wird hier auf den Rechtscode Bezug genommen, indem für einen technischen Standard ein fundamentales Verständnis von „Privacy“ oder „informationeller Selbstbestimmung“ festlegt wird: Selbstbestimmung des Internetnutzers über die Verwendung der datenschutzsensitiven Cookies, ermöglicht durch die Übersetzung dieses Prinzips in Technik.

Ähnliche, jedoch in der juristischen Diskussion bislang weniger beachtete Beispiele lassen sich auch für das Problem sogenannter Location Based Services im Mobilfunkbereich finden. So hat die IETF mit der Geopriv working group die Arbeit für die Entwicklung eines Standards auf den Weg gebracht, der datenschutzrechtlich relevante Fragen in Location Based Services adressiert. ${ }^{80}$ Eine weitere Standardisierungsorganisation, das Open Geospatial Consortium (OGC), unterhält eine Working Group zu Geospatial Rights Management. Der Einsicht geschuldet, dass „the obstacles preventing greater use of geospatial data and services are increasingly policy related, not technical”, arbeitet die Working Group an der Entwicklung von Standards, welche die Einbindung divergierender privacy policies in Location Based Services erlauben. ${ }^{81}$ Das W3C hat im Oktober 2007 eine Policy Languages Interest Group gegründet, die sich mit der Interoperabilität der verschiedenen derzeit erhältlichen Privacy Protokolle beschäftigen soll. ${ }^{82}$ Festzuhalten bleibt, dass im Bereich der Entwicklung technischer Internetstandards vermehrt Rechtskommunikationen auftauchen.

\section{b) Sekundäre Normierungen}

In einem zweiten Schritt sind diese rechtlichen Kommunikationen daraufhin zu untersuchen, ob ihr Entstehungsprozess durch sekundäre Normen strukturiert ist. Die Statuten der - hier allein in den Blick genommenen - Standardisierungsgremien IETF und W3C sehen zwar ausführliche Verfahrensvorschriften für die Entwicklung eines Standards vor, die dem Gesetzgebungsverfahren einer nationalstaatlichen Verfassung durchaus nicht unähnlich sind. Es erscheint aber äußerst fraglich, ob man sie aber als sekundäre Normierungen qualifizieren kann. Eine pragmatische Sichtweise könnte die in den Statuten normierten Verfahrensvorschriften lediglich als Erfordernis ansehen, das der Interoperabilität und sprechende RFC 4079 ist erhältlich unter http://www.rfc-ref.org/RFC-TEXTS/4079/index.html (abgerufen: 25.3.2009).

81 Siehe http://www.opengeospatial.org/ogc/markets-technologies/georm (abgerufen: 25.3.2009).

82 Siehe http://www.w3.org/Privacy/ (abgerufen: 25.3.2009). 
der Effektivität der entwickelten Standards geschuldet ist: Um möglichst umfas-

send den im Netz diffus vorhandenen Sachverstand von Technikexperten nutzen zu können, muss die Beteiligung an der Diskussion um die Entwicklung eines Standards offen sein; um angesichts der Partizipationsmöglichkeit vieler gleichwohl die Effektivität des Prozesses gewährleisten zu können, müssen hierarchische Entscheidungsstrukturen kodifiziert werden, die den Entwicklungsprozess zum Abschluss bringen können. Auf der anderen Seite ist jedoch nicht zu übersehen, dass genau diese Verfahrensvorschriften auch Einfluss auf die mitlaufende rechtliche Kommunikation im Sinne einer sekundären Normierung ihrer Entstehung haben, indem sie überhaupt erst Artikulationschancen für Rechtsthemen eröffnen.

Vor diesem Hintergrund ist die Entwicklung von technischen Internetstandards auch aus einer rechtspluralistischen Perspektive heraus noch weit davon entfernt, im gleichen Maße wie etwa die lex mercatoria als operativ geschlossenes (autonomes) Recht angesehen werden zu können. Zwar ist nicht zu übersehen, dass es vereinzelte Kommunikationen nach dem binären Rechtscode gibt, im Unterschied zu Einrichtungen wie den ICANN-Schiedspanel's existiert aber in diesem Bereich kein Forum, anhand dessen sich die inkrementelle Herausbildung von Prozessen der sekundären Normierung ablesen lassen könnte..$^{83}$ Die vorhandenen Rechtskommunikationen im Bereich der technischen Internetstandardisierung sind gerade aufgrund der Abwesenheit von institutionalisierten sekundären Normierungen in noch stärkerem Maße als die lex mercatoria durch strukturelle Korruption anderer Sozialbereiche gekennzeichnet, was den ungefilterten Einfluss von Partikularinteressen (insbesondere der Wirtschaft) begünstigt.

\section{Transnationales Datenschutzrecht als Kollisionsrecht}

Was folgt daraus für einen regulatorischen Ansatz, der an solche privaten Normierungen auf transnationaler Ebene anschließen will? Ein Weg wäre, den technischen Internetstandards den Segen der nationalen Behörden durch Zertifizierungen im Rahmen eines Datenschutzaudits zu geben. ${ }^{84}$ Damit fängt man sich jedoch das Problem ein, dass staatlicher Gesetzgebung und ihrem Vollzug durch nationale Datenschutzbehörden (oder durch Kooperation nationaler Behörden) ${ }^{85}$ weder auf globaler noch auf nationaler Ebene ein Wissensvorsprung zuzusprechen ist und die Entwicklung technischer Standards nicht aus einer übergeordneten staatlichen Perspektive durch Zertifizierung regulierbar ist. Die Abhängigkeit des Staates von Expertenwissen ist im nationalen Technikrecht mit seinen Verweisen auf den Stand der Technik noch weitestgehend in tradierte rechtstheoretische Modelle integriert worden. ${ }^{86}$ Für die Entwicklung technischer Standards auf globaler Ebene fehlt es an einer übergeordneten politischen Instanz, die diese Abhängigkeiten „von oben“ in ein hierarchisches Modell der Einheit der Rechtsordnung einbinden könnte.

Teubners Rechtspluralismus lebt insbesondere von der Existenz von Konfliktlösungsinstanzen, die privat gesetzten Rechtsnormen einer Mikrovariation unter sekundären Beobachtungen anhand des binären Rechtscodes unterziehen, so etwa für die Lex digitalis des Internets, die er in erster Linie anhand der ICANN-Schiedspanels zu plausibilisieren versucht, siehe nur Teubner (Fn. 75); Karavas/Teubner, http://www.CompanyNameSucks.com: Drittwirkung der Grundrechte gegenüber „Privaten“ im autonomen Recht des Internet?, in: Ladeur (Hrsg.), Innovationsoffene Regulierung des Internet, 2003, S. $249 \mathrm{ff}$.

$84 \mathrm{Zu}$ kurz auch Bennett/Raab, The governance of privacy: policy instruments in global perspective, 2. Auflage 2006, S. 229.

85 Dazu Möllers, Transnationale Behördenkooperation. Verfassungs- und völkerrechtliche Probleme transnationaler administrativer Standardsetzung, ZaöRV 65 (2005), $351 \mathrm{ff.}$

Siehe oben C.I. mit weiteren Nachweisen. 
Wir hatten stattdessen angekündigt, ein transnationales Verwaltungsrecht als Kollisionsrecht zu entwickeln. Das bedarf insofern der Erläuterung, als mit der tradierten kollisionsrechtlichen Semantik nur die Abgrenzung der Geltungs- und Anwendungsbereiche von nationalstaatlichen Verwaltungsrechtsordnungen geleistet wird. Wir schließen hier aber an neuere kollisionstheoretische Arbeiten an, die unter expliziter Aufgabe eines nationalstaatlich fixierten Rechtsbegriffs Kollisionslagen in einem umfassenderen Sinn auf Konflikte zwischen Teilrechtsordnungen beziehen, die an den Kontaktzonen des Rechts von autonomen Sozialbereichen quer zur segmentären Differenzierung in Nationalstaaten hervorgebracht werden. ${ }^{87}$ Statt hierarchischer Normeneinheit auf globaler Ebene für alle Bereich des Rechts verfolgen solche Ansätze die nachbarschaftlich-heterarchische Koordination solcher Teilrechtsordnungen. ${ }^{88}$ Die Normerzeugung durch Private auf internationaler Ebene verschleift sich im „Internationalen Verwaltungsrecht “89 derart, dass nicht „zwischen internationalen Normen und Standards, transnationalen Prozeduren der Normerzeugung außerhalb eines organisierten internationalem Rechtsregimes und der nationalen Verwaltungsebene"90 getrennt werden kann. „Transnationales Verwaltungsrecht“ als Kollisionsrecht hat dann dafür zu sorgen, Konflikte zwischen staatlichen Verwaltungsordnungen und nicht-staatlichen Normierungsprozessen kommensurabel zu machen, statt eine dem Gedanken der Einheit der Rechtsordnung folgende Normenhierarchie herzustellen. ${ }^{91}$

Damit überwinden wir auch Forderungen nach einer Ablösung der kollisionsrechtlichen Semantik des traditionellen internationalen Verwaltungsrechts und einer Neuformulierung als im Völkerrecht begründetes Verwaltungsrecht. ${ }^{92}$ Zwar löst man sich so von der Fixierung auf die Kollision staatlicher Rechtsordnungen, ${ }^{93}$ allerdings ist die Engführung auf das Völkerrecht für unsere Zwecke wenig brauchbar. Probleme entstehen nicht nur dadurch, dass die nationalen Verwaltungen vermehrt für die Kooperation mit anderen Verwaltungen durchlässig werden und sich dadurch den klassischen rechtsstaatlichen Bindungen zu entziehen drohen; ${ }^{94}$ technische Standardisierung ist kein Problem der transnationalen Kooperation nationaler Datenschutzbehörden, die auf globaler Eben gemeinsame Datenschutzstandards zu finden versuchen. Viel drängender ist die Verselbstständigung eines Technikdiskurses, der einerseits damit beginnt, eigenständige Normierungen aus sich herauszutreiben, und dessen Universalisierungsanspruch andererseits massive Auswirkungen auf andere Sozialbereiche hat.

\section{Wechselseitige Beeinflussung}

Diese Perspektive ermöglicht es, ein Kollisionsrecht zu formulieren, das „,in dieser Konstellation die wechselseitigen Zuständigkeiten nicht mehr primär von-Lescano/Teubner (Fn. 46); Fischer-Lescano (Fn. 41), 373. Grundlegend Wietholter, Zum Fortbildungsrecht der (richterlichen) Rechtsfortbildung. Fragen eines lesenden Recht-Fertigungslehrers, KritV 1988, $1 \mathrm{ff}$.

88 Fischer-Lescano/Teubner (Fn. 74), $37 \mathrm{ff}$.

89 Möllers/Voßkuhle/Walter (Hrsg.) (Fn. 54).

90 Ladeur (Fn. 58), 388.

91 Fischer-Lescano/Teubner (Fn. 46), S. 68. 92 Schmidt-Aßmann, Die Herausforderung der Verwaltungsrechtswissenschaft durch die Internationali-

93 Fischer-Lescano (Fn. 41), 373.

94 Schmidt-Aßmann (Fn. 92), 324. 
einander abgrenzt, sondern füreinander durchlässig hält, aber zugleich auch die Grenzen dieser Durchlässigkeit beobachtet, justiert und festlegt. “95 Damit haben wir ein Konzept der wechselseitigen Beeinflussung formuliert, dass für beide Seiten sowohl ein kognitives als auch ein normatives Element enthält.

Aus der Perspektive des nationalen Rechts bedeutet „Füreinander durchlässig halten“ zunächst, sich viel stärker für Normierungen im privaten Bereich zu öffnen. ${ }^{96}$ Der Grund dafür ist, dass die Normbildung in den Sozialbereichen immer eng mit dessen Eigenlogik verknüpft ist. ${ }^{97}$ Bei der inkrementellen Herausbildung von Rechtsnormen im Bereich der Internetstandards zeigt sich das daran, dass die Erfordernisse der Interoperabilität und der Anschlussfähigkeit für zukünftige Entwicklungen in einem heterarchisch organisierten Computernetzwerk immer auf eigentümliche Weise mit den auftauchenden Normierungen verknüpft sind. Ähnlich dem Gefahrenbegriff im Polizeirecht, der an gesellschaftliches Wissen anknüpft und sich so schon vorhandene Erfahrung zunutze macht, ${ }^{98}$ muss Rechtsnormbildung im Bereich der technischen Internetstandardisierung parasitär an vorhandenes Wissen anknüpfen, das nicht aus seinem praktischen Anwendungskontext herausgelöst werden kann. ${ }^{99}$ Wie man in standortbasierte Kommunikationsprotokolle Fragen des Datenschutzes einbinden kann, ist nur im Vollzug der Entwicklung eines Standards thematisierbar (z.B. Geopriv). Dadurch kommt es zu einer engen Verschleifung von Rechtsnormen und der technischen Logik von Internetstandards. Sobald sich also datenschutzrechtliche Probleme auf den unteren, durch technische Standards determinierten Ebenen der Internetkommunikation stellen (etwa die Verwendung von Cookies), könnte der Anspruch der nationalen Datenschutzgesetze zurückgenommen werden, soweit solche Probleme schon auf der Ebene der Entwicklung des Standards thematisiert wurden. Der Vorteil für eine derartige Regulierungsstrategie liegt darin, dass sich so vor allem der ansonsten schwer institutionalisierbare Sachverstand zunutze machen lässt.

Gleichzeitig, und sozusagen im Austausch für die Anerkennung ihrer Verbindlichkeit, könnten rechtsstaatlichen Mindestanforderungen an die transnationalen Normbildungsprozesse über eine Art „Solange“-Formel rechtlich institutionalisiert und damit die Grenzen dieses Konzepts der Wechselseitigkeit festgelegt werden. Anerkennung darf dann nur um den Preis erfolgen, gewisse rechtsstaatliche Mindeststandards bei der Einbindung rechtlicher Themen in die Entwicklung technischer Standards zu beachten. Die Kollisionen des Technikdiskurses mit anderen Rationalitäten nötigen weiterhin als normative Forderung dazu, Mechanismen der Reflexion über destruktive Tendenzen innerhalb dieses Diskurses zu institutionalisieren. Hier bietet sich mit dem Institut der Technikfolgenabschätzung ${ }^{100}$ ein erster brauchbarer Ansatz, der dazu nötigen würde, schon bei der Entwicklung eines Standards Auswirkungen auf die informationelle Selbstbestimmung zu thematisieren.

Umgekehrt kann sich der Sozialbereich Technik die insbesondere legitimatorischen Leistungen des hochgradig institutionalisierten Rechts der Nationalstaaten zunutze machen. Eine Kollisionsregel würde nämlich den erforderlichen Anreiz

96 Für das Datenschutzrecht siehe nur Vesting, Das Internet und die Notwendigkeit der Transformation des Datenschutzes, in: Ladeur (Hrsg.), Innovationsoffene Regulierung des Internet, 2003, S. 155 ff.

97 Teubner (Fn. 72), 263; Herberg (Fn. 41), 77 ff.

98 Ladeur, Das Umweltrecht der Wissensgesellschaft: von der Gefahrenabwehr zum Risikomanagement, 1995, S. $11 \mathrm{ff}$.

99 Ladeur (Fn. 58), 389.

100 Dazu die Tätigkeitsberichte des Büros für Technikfolgenabschätzung beim Deutschen Bundestag, abrufbar unter http://www.tab.fzk.de/de/arbeitsberichte.htm (abgerufen: 25.3.2009). 
bieten, die bislang noch vereinzelt bleibende Thematisierung rechtlicher Themen in den Standardisierungsorganisationen stärker institutionell abzustützen. Die bislang noch nicht geschlossen (autonom) operierende Teilrechtsordnung des Standardisierungsbereichs würde so in ihrem Entwicklungsprozess stärker autonomisiert und gegenüber korrumpierenden Einflüssen anderer Sozialbereiche widerstandsfähiger gemacht. Das erst setzt die Kapazitäten innerhalb des Sozialbereichs Technik frei, deren primäre Nutzung - neben Mechanismen der internationalen Politik - die einzig erfolgversprechende Strategie einer Regulierung sein kann. Belässt man die Entwicklungstätigkeit der Technikdesigner im Schatten des nationalstaatlichen Rechts, dann bleibt diese Entwicklungstätigkeit auch „nur“ Technik, trotz all der massiven normativen Konsequenzen für ihre Umwelten. Hebt man sie aber ans Licht und anerkennt die rechtliche Relevanz ihrer Normierungstätigkeiten, versetzt man ihre gesellschaftlichen Umwelten erst in die Lage, gewisse rechtsstaatliche Standards und Mindestanforderungen des Ausgleichs widerstreitender Interessen an sie zu stellen, die immer mit dem Begriff des Rechts verbunden sind.

Ein solches Konzept, das transnationales Datenschutzrecht als Kollisionsrecht von nationalen Verwaltungsordnungen mit privaten Normierungstätigkeiten auf globaler Ebene rechtlich zu institutionalisieren versucht, geht über die monetäre Förderung der Entwicklung von Privacy Enhancing Technologies durch den Staat hinaus. Der entscheidende Vorteil liegt gerade in der rechtlichen Institutionalisierung dieser Kooperationsbeziehung, die wechselseitige Lernprozesse fördert und so direkter an das nötige, durch den Sozialbereich selbst hervorgebrachte „Regulierungswissen“ anknüpfen kann.

\section{Korruption und Zivilrecht}

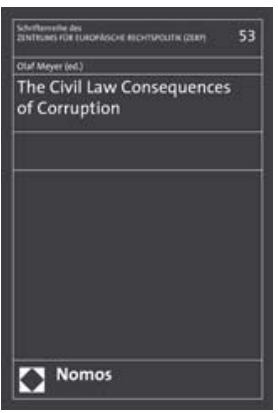

\section{The Civil Law Consequences of Corruption} Herausgegeben von Dr. Olaf Meyer, M.St. (Oxon.)

2009, 280 S., brosch., 68,- $€$, ISBN 978-3-8329-4496-4

(Schriftenreihe des Zentrums für Europäische Rechtspolitik an der Universität Bremen (ZERP), Bd. 53)

Zum ersten Mal werden in diesem Buch die Möglichkeiten diskutiert, Zivilrecht gezielt als Waffe gegen Korruption einzusetzen. Im Mittelpunkt stehen dabei die Fragen nach der Vertragswirksamkeit, der Gewinnabschöpfung und dem Schadensersatz. Neben 10 Länderberichten zur Lage in Europa und den USA kommen auch Vertreter internationaler Organisationen zu Wort.

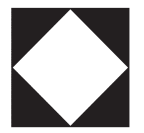

Nomos

Bitte bestellen Sie im Buchhandel oder versandkostenfrei unter $\downarrow$ www.nomos-shop.de 